\title{
The Role of Cardiac Biomarkers as Predictors of In-hospital Mortality among COVID patients in a Tertiary Hospital in Metro Manila, Philippines
}

\author{
Renato Ong Jr., RMT, MD, FPCP, ISMPP, ${ }^{1}$ Chadwick Chacon, MD, FPCP, ${ }^{1}$ Saturnino Javier, MD, FPCP, FPCC \\ 'Section of Cardiology, Makati Medical Center, Philippines \\ Contact information: r.ongjrmd@gmail.com
}

\begin{abstract}
\section{BACKGROUND}

Coronavirus disease 2019 (COVID-19) has been declared a global pandemic. The overwhelming volume of confirmed cases makes prevention and control a challenge. Despite this, numerous knowledge gaps remain in the diagnosis, management and prognostication of this novel coronavirus infection.
\end{abstract}

\section{METHODS}

This retrospective cohort study included patients with polymerase chain reaction confirmed COVID-19. Binary logistic regression was used to determine the association between the cardiac biomarkers and in-hospital mortality. ROC, AUC and cutoff analyses were used to determine optimal cutoff values for the cardiac biomarkers.

\section{RESULTS}

Out of 224 rRT-PCR confirmed cases, 90 subjects with a complete panel of cardiac biomarkers were included in this study. Sixtysix $(77.6 \%)$ subjects survived, five were still admitted while 19 (22.4\%) expired. Logistic regression analysis showed that creatine kinase and hsTnl were independently associated with inhospital mortality (OR, 4.103 [95\% Cl, 1.241-13.563], $\mathrm{p}=0.021$; and $\mathrm{OR}, 7.899$ [95\% Cl, 2.430-25.675], $\mathrm{p}=0.001$, respectively). ROC curve analysis showed that hsTnl was a good predictor for in-hospital mortality (AUC, 0.829 [95\% Cl, 0.735-0.923], p $=<0.001)$. Optimal cutoff point derived from the ROC curve for hsTnl was $0.010 \mathrm{ng} / \mathrm{ml}(\mathrm{J}, 0.574)$ with a sensitivity of $84 \%$ (TPR, 0.842 [95\% Cl, 0.604-0.966]), specificity of 73\% (TNR, 0.732 [95\% $\mathrm{Cl}, 0.614-0.386])$, an adjusted negative predictive value of $99 \%$ (Known prevalence*adjusted NPV, 0.989), a positive likelihood ratio of 20\% (LR+, 3.147 [95\% Cl, 2.044-4.844]).

\section{CONCLUSION}

High sensitivity troponin I level was a good tool in significantly predicting in-hospital mortality.

\section{INTRODUCTION}

After coronavirus disease 2019 (COVID-19) has been declared a pandemic, there are now 6,702,699 COVID-19 cases worldwide, with a computed prevalence rate of 83.8 per 100,000 and incidence rate of 38.2 per $100,000 .^{1}$ As of May 2020, the Philippines ranks $39^{\text {th }}$ out of the 215 countries in the number of cases with a case fatality rate of $4.4 \%$. As the viral pandemic decimates lives and paralyzes economies, numerous knowledge gaps remain in the diagnosis, management and prognostication of this novel coronavirus infection.
This study aimed to determine the role of cardiac biomarkers as predictor of in-hospital mortality among nucleic acid amplification test (real-time reverse transcriptase polymerase chain reaction or RT-PCR) positive patients for SARS-CoV-19 in Makati Medical Center (MMC), a 600-bed capacity tertiary facility in Makati City, Metro Manila, Philippines which was one of the frontline institutions in the National Capital Region of the Philippines which battled the first wave of COVID-19 starting in March 2020.

\section{METHODS}

This was a retrospective cohort of consecutive rRT-PCR seropositive COVID-19 patients, aged 18-90 years, admitted at MMC from February 1 to April 30, 2020. Those who stayed in the hospital for less than six hours and those with no determination of cardiac biomarkers were excluded. The patients were grouped into non-severe and severe categories (the severe and critical cases were grouped all together). Further, each category was subcategorized as to the final clinical outcome - survivors and non-survivors.

All data were extracted from electronic medical records and patient charts: demographic data, i.e., age, sex, occupation, body mass index, exposure and smoking history; clinical data, i.e., signs or symptoms upon presentation, oxygen saturations, and supplementations given, pre-existing comorbidities, complications, and clinical outcome.

The results of the following laboratory parameters were noted at baseline contact: complete blood count, platelet count, creatinine, sodium, potassium, aspartate aminotransaminase (AST), alanine aminotransferase (ALT), ferritin, C-reactive protein (CRP), procalcitonin, lactate dehydrogenase (LDH), hs troponin I (hsTnl), total creatine kinase (tCK) and creatine kinase-MB (CK$\mathrm{MB}$ ) isoenzyme. Data were entered into a password-protected computerized database, cross-checked and encrypted.

The diagnosis was based on the World Health Organization (WHO) interim guidelines. ${ }^{2} \mathrm{~A}$ confirmed case of COVID-19 was defined as a positive result on the rRT-PCR assay of nasopharyngeal and oropharyngeal swab specimens. Nonsevere cases are patients with uncomplicated upper respiratory tract infection with non-specific symptoms such as fever, fatigue, cough (with or without sputum production), respiratory rate of fewer than 30 breaths/min, and SPO2 more than 93\% at room air; severe cases are patients with severe pneumonia with either one of the following: dyspnea, the respiratory rate more than 30 breaths/min, sPO2 less than or equal to $93 \%$ at room air, the partial pressure of arterial oxygen to fraction of inspired 
oxygen (P/F) ratio of less than 300 , sepsis or septic shock, and lung infiltrates more than 50\% within 24 to 48 hours; and critical cases are those who are in respiratory failure, necessitating the need for mechanical intubation, septic shock, and/or multiorgan dysfunction or failure..$^{3,4}$ The primary composite end-point was defined as an event where patients required either intensive care monitoring, mechanical intubation and/or death.

The following in-hospital cardiovascular and other complications were defined as follows: acute heart failure syndrome was defined as a change in heart failure signs and symptoms requiring urgent therapy; significant arrhythmia pertained to any of the following, i.e., ventricular tachycardia, atrial/ventricular fibrillation, and ectopic atrial rhythm; vasopressor requiring shock defined as a hemodynamic instability requiring inotropic use; acute respiratory distress syndrome was defined according to the Berlin definition of $2012 ;{ }^{5}$ acute kidney injury was defined according to the Kidney Disease Improving Global Outcomes guidelines. ${ }^{6}$

This study was conducted in compliance with all local and national regulations and guidelines and in accordance with the International Conference on Harmonization and Good Clinical Practice. The protocol of this study was reviewed and approved by the MMC Institutional Review Board. The privacy and confidentiality of data were maintained in compliance with the Philippine Data Privacy Act of 2012. The authors had no conflicts of interest in the study.

\section{STATISTICAL ANALYSIS}

Categorical variables were shown as counts and percentages, and continuous variables as medians and interquartile ranges (IQR). Mann-Whitney $U$ test was performed for continuous variables, and the $\boldsymbol{\chi}^{2}$ test and Fisher exact test were used for categorical variables as appropriate. The Spearman rho for nonparametric measures was used to measure the degree of association of the cardiac biomarkers with the primary composite endpoint and the disease severity. Kruskal-Wallis $\mathrm{H}$ test was used to compare across groups. No imputation was made for missing data.

Binary logistic regression analysis was used to determine the association of cardiac biomarkers with mortality. The odds ratio (OR) along with the 95\% confidence interval $(\mathrm{Cl})$ were reported A $p$ value of less than 0.05 was regarded as statistically significant. All statistical analyses were performed using IBM SPSS Statistics 22 for Windows, and NCSS 2020 Statistical Software (2020). NCSS, LLC.

\section{RESULTS}

After carefully reviewing 224 records of confirmed COVID-19 patients for completion of data, 90 patients with cardiac biomarker determinations were included in this study. In the non-severe category, there were 60 cases, 59 (98.3\%) survived and one $(1.7 \%)$ died; while in the severe category, there were 30 cases of which there were $12(40 \%)$ survivors and $18(60 \%)$ non-survivors.

The median age for all patients was 57 years (IQR, 47-67 years) Around seven $(40 \%)$ of the non-survivors in the severe category were aged 50-59 years, while two (11\%) were aged above 80 years. There were significantly more older individuals who died than those who survived. Majority of the patients, 62 (68.9\%) were males, $39(62.9 \%)$ in the non-severe, and $23(37.1 \%)$ in the severe category. A few, 7 (7.8\%) were healthcare workers (Table 1).

\section{CLINICAL PROFILE UPON PRESENTATION AND PRE- EXISTING CO-MORBID CONDITIONS}

The three most common presenting symptoms were fever (65, $75.6 \%)$, cough $(48,55.8 \%)$ and sputum production (18, 20.9\%) as shown in Figure 1. All patients in the non-severe group (100\%; $p=0.013$ ) and more than $90 \%$ of the non-survivors in the severe group had cough $(p=0.003)$.

\section{Figure 1: Distribution of Symptoms Presented by Patients at the Emergency Department.}

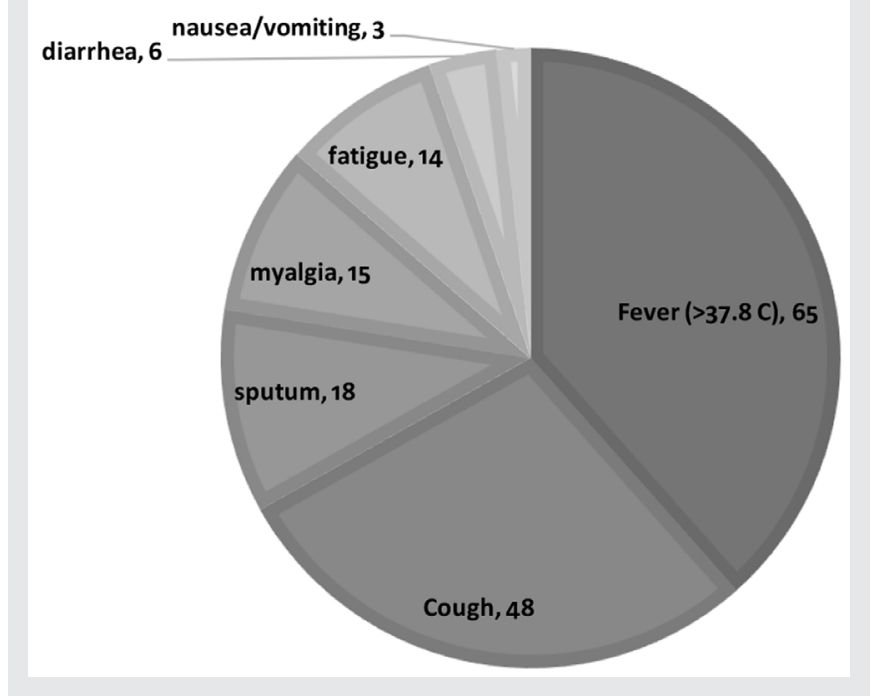

The three most common co-morbid conditions were hypertension $(61,67.8 \%)$, diabetes mellitus $(30,33.3 \%)$ and bronchial asthma $(15,16.7 \%)$ as shown in Table 2. Fourteen (15.6\%) had chronic cardiac disease, and ten (11.1\%) had chronic kidney disease.

\section{LABORATORY FINDINGS}

As shown in Table 3, the median lymphocyte count of the non-survivors in the severe category was significantly lower than those who survived $\left(1,250\right.$ cells $/ \mathrm{mm}^{3}$ [IQR, 850-1,775] vs. 2,000 cells $/ \mathrm{mm}^{3}$ [IQR, 1,600-2,700], $\left.p=0.001\right)$. No statistically significant difference for white blood cell count, platelet count and hemoglobin level in both categories was found.

The median lactate dehydrogenase levels of the survivors, 272.8 $\mathrm{U} /$ /iter and 323.0 $\mathrm{U} /$ /iter, were significantly lower than those of the non-survivors, 640.91 U/liter and 391.3 U/liter [IQR, 345.3-514.4], for both the non-severe and the severe groups $(p<0.001$, and $p$ $<0.001$, respectively)

In the severe category, the median C-reactive protein levels was significantly higher among the non-survivors (13.9 mg/liter [IQR, 11.5-19.43] vs. $9.84 \mathrm{mg} /$ liter [IQR, 4.88-16.25], $p<0.001$ ) Also, the median ferritin levels among those who survived was significantly lower relative to those who did not $(1,646.3 \mu /$ liter [IQR, 926.2-2,446.7] vs. 1,859.2 $\mu /$ liter [IQR, 1,246-2,671.5], $p=$ $0.0010)$

The procalcitonin levels was significantly higher among the nonsurvivors relative to those who survived across both severe $(0.60$ 


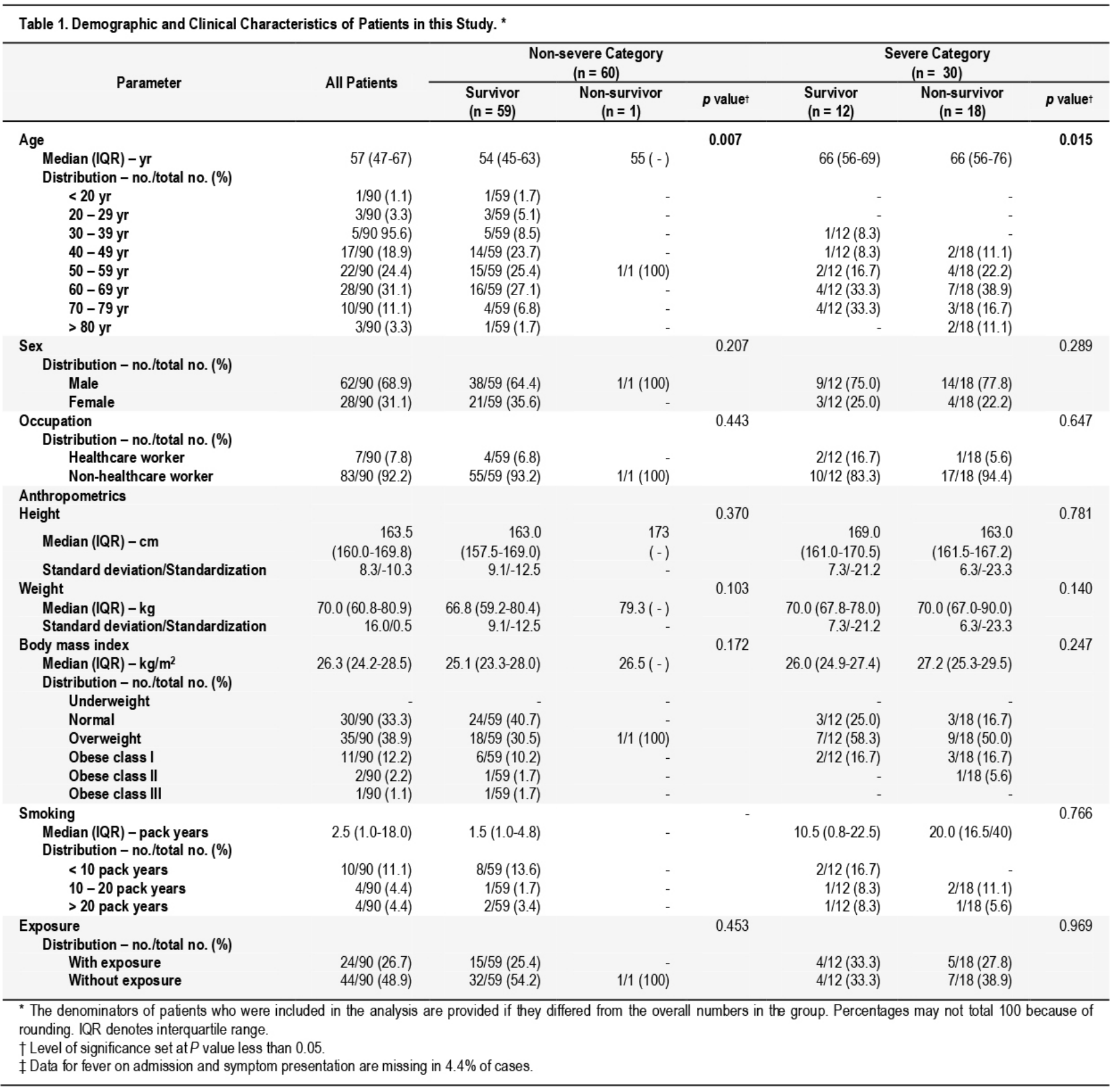




\begin{tabular}{|c|c|c|c|c|c|c|c|}
\hline \multirow{2}{*}{ Parameter } & \multirow{2}{*}{ All Patients } & \multicolumn{2}{|c|}{$\begin{array}{c}\text { Non-severe Category } \\
(\mathrm{n}=60)\end{array}$} & \multicolumn{4}{|c|}{$\begin{array}{c}\text { Severe Category } \\
(\mathrm{n}=30)\end{array}$} \\
\hline & & $\begin{array}{l}\text { Survivor } \\
(n=59)\end{array}$ & $\begin{array}{l}\text { Non-survivor } \\
(n=1)\end{array}$ & $p$ value & $\begin{array}{l}\text { Survivor } \\
(n=12)\end{array}$ & $\begin{array}{c}\begin{array}{c}\text { Non-survivor } \\
(\mathrm{n}=18)\end{array} \\
\end{array}$ & $p$ value \\
\hline \multicolumn{8}{|l|}{ Temperature at the Emergency Room } \\
\hline Median (IQR) - ${ }^{\circ} \mathrm{C}$ & $\begin{array}{r}37.1 \\
(36.5-37.8)\end{array}$ & $\begin{array}{r}37.0 \\
(36-3-378)\end{array}$ & $\begin{array}{r}37.3 \\
(-)\end{array}$ & & $\begin{array}{r}37.5 \\
(370-37.8)\end{array}$ & $\begin{array}{r}37.7 \\
(370-3799\end{array}$ & \\
\hline Distribution - no./total no. (\%) & & & & & & & \\
\hline$<37.5^{\circ} \mathrm{C}$ & $54 / 86(62.8)$ & $41 / 59(69.5)$ & $1 / 1(100)$ & & $5 / 10(50.0)$ & $7 / 16(43.8)$ & \\
\hline $37.5-38.0^{\circ} \mathrm{C}$ & $17 / 86(19.8)$ & $8 / 59(13.6)$ & & & $4 / 10(40.0)$ & $6 / 16(37.5)$ & \\
\hline $38.1-39.0^{\circ} \mathrm{C}$ & $10 / 86(11.6)$ & $7 / 59(11.9)$ & - & & $1 / 10(10.0)$ & $2 / 16(12.5)$ & \\
\hline$>39^{\circ} \mathrm{C}$ & $5 / 86(5.8)$ & $3 / 59(5.1)$ & - & & $1 / 10(10.0)$ & $1 / 16(6.3)$ & \\
\hline Fever & $65 / 86(75.6)$ & $47 / 59(79.7)$ & - & 0.022 & $6 / 10(60.0)$ & $11 / 16(68.8)$ & 0.245 \\
\hline Cough & $48 / 86(55.8)$ & $59 / 59(44.1)$ & - & 0.013 & $6 / 10(60.0)$ & $15 / 16(93.8)$ & 0.003 \\
\hline Sputum production & $18 / 86(20.9)$ & $13 / 59(22.0)$ & & 0.906 & & $5 / 16(31.3)$ & 0.441 \\
\hline Myalgia & $15 / 86(17.4)$ & $8 / 59(13.6)$ & $1 / 1(100)$ & 0.203 & $2 / 10(20.0)$ & $5 / 16(31.3)$ & 0.206 \\
\hline Fatigue & $14 / 86(16.3)$ & $10 / 59(16.9)$ & & 0.257 & $2 / 10(20.0)$ & $1 / 16(6.3)$ & 0.498 \\
\hline Diarrhea & $6 / 86(7.0)$ & $1 / 59(1.7)$ & - & 0.183 & $2 / 10(20.0)$ & $3 / 16(18.8)$ & 0.074 \\
\hline Nausea/vomiting & $3 / 86(3.0)$ & $1 / 59(1.7)$ & - & 0.113 & & $2 / 16(25.0)$ & 0.051 \\
\hline \multirow{2}{*}{\multicolumn{8}{|c|}{$\begin{array}{l}\text { Co-existing disorders } \\
\text { Distribution - no./total no. (\%) }\end{array}$}} \\
\hline & & & & & & & \\
\hline Diabetes mellitus & $30 / 90(33.3)$ & $16 / 59(27.1)$ & - & 0.131 & $7 / 12(58.3)$ & $7 / 118(38.9)$ & 0.716 \\
\hline Hypertension & $61 / 90(67.8)$ & $36 / 59(61.0)$ & $1 / 1(100)$ & 0.258 & $8 / 12(66.7)$ & $16 / 18(88.9)$ & 0.055 \\
\hline Chronic cardiac disease & $14 / 90(15.6)$ & $6 / 59(10.2)$ & . & 0.138 & $4 / 12(33.3)$ & $4 / 18(22.2)$ & 0.459 \\
\hline Cerebrovascular disease & $3 / 90(3.3)$ & $1 / 59(1.7)$ & - & & $1 / 12(8.3)$ & $1 / 18(5.6)$ & 0.600 \\
\hline Chronic renal disease & $10 / 90(11.1)$ & $4 / 59(6.8)$ & - & 0.240 & $2 / 12(16.7)$ & $4 / 18(22.2)$ & 0.123 \\
\hline Chronic liver disease & $2 / 90(2.2)$ & $1 / 59(1.7)$ & - & 0.391 & & $1 / 18(5.6)$ & 0.462 \\
\hline Malignancy $\ddagger$ & $2190(2.2)$ & $1 / 59(1.7)$ & - & 0.391 & - & & \\
\hline Hematologic disease & $1 / 90(1.1)$ & $1 / 59(1.7)$ & - & 0.546 & & & \\
\hline Chronic respiratory diseases & $2 / 90(2.2)$ & & - & & $1 / 12(8.3)$ & $1 / 18(5.6)$ & 0.314 \\
\hline $\begin{array}{l}\text { Bronchial asthma } \\
\text { Buscos }\end{array}$ & $15 / 90(16.7)$ & $10 / 59(16.9)$ & - & 0.525 & $2 / 12(16.7)$ & $3 / 18(16.7)$ & 0.909 \\
\hline Thyroid pathology & $2 / 90(2.2)$ & $2159(3.4)$ & - & 0.324 & & & \\
\hline Dyslipidemia & $6 / 90(6.7)$ & $5 / 59(8.5)$ & - & 0.569 & - & $1 / 18(5.6)$ & \\
\hline Any $\pi$ & $20 / 90(22.2)$ & $20 / 59(33.9)$ & - & 0.577 & & $6 / 18(33.3)$ & 0.268 \\
\hline
\end{tabular}

* The denominators of patients who were included in the analysis are provided if they differed from the overall numbers in the group. Percentages may not total 100 because of rounding. IQR denotes interquartile range. $P$ value less than 0.05 is considered significant.

$\dagger$ Data for fever on admission and symptom presentation are missing in $4.4 \%$ of cases.

$\ddagger$ Included in this category is any type of cancer.

$\S$ Chronic respiratory disease encompasses physician-diagnosed chronic obstructive pulmonary disease, bronchiectasis, etc.

ๆ Other conditions not belonging to the major categories listed, includes: osteoporosis, hyperuricemia, gouty arthritis, impared fasting glucose, gastroesophageal reflux disease

irritable bowel syndrome, osteoarthritis, and benign prostatic hyperplasia

** The primary composite end point was admission to an intensive care unit, the use of mechanical ventilation, or death.

t† Data for disposition were missing in $5.5 \% \%$ of cases

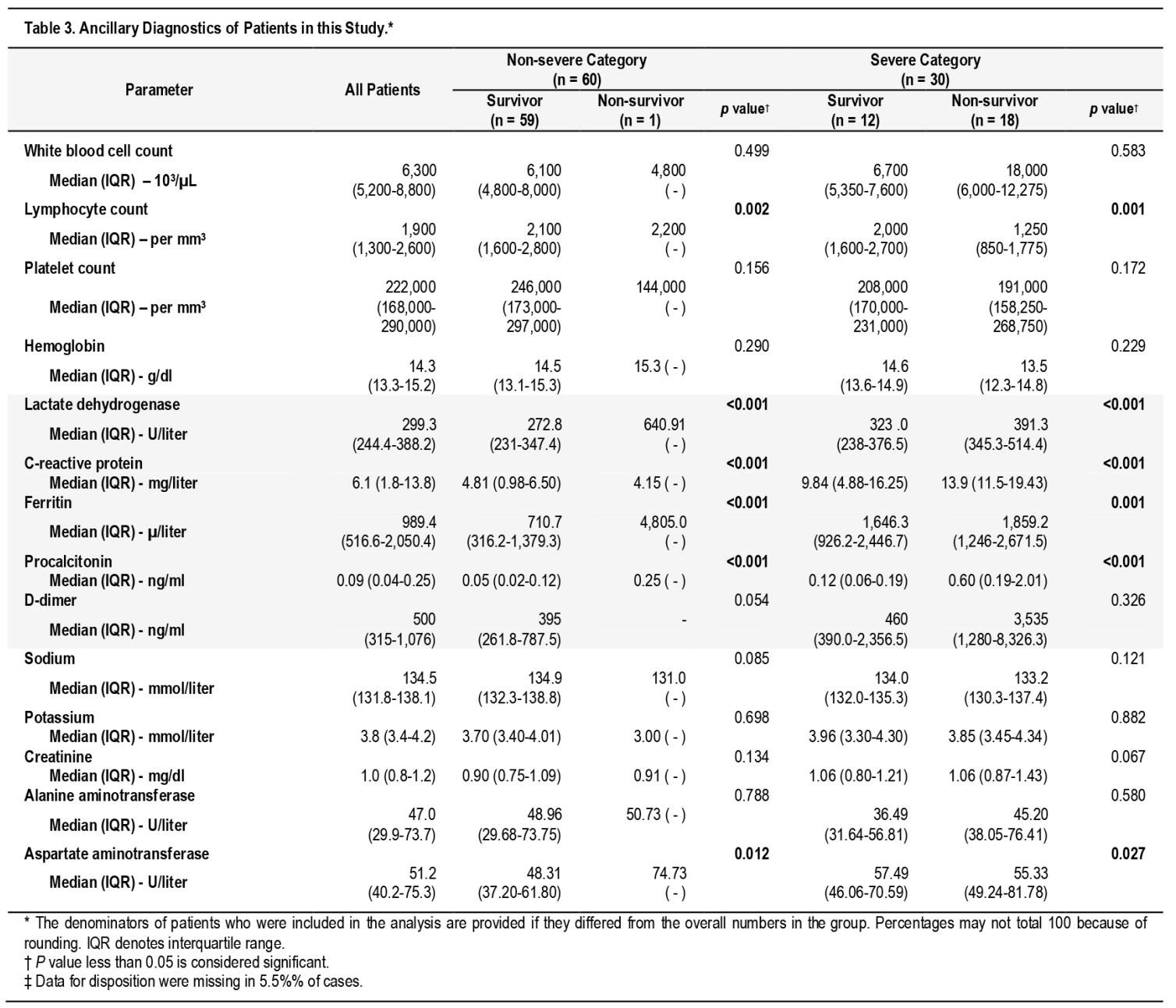




\begin{tabular}{|c|c|c|c|c|c|c|c|}
\hline \multirow{2}{*}{ Parameter } & \multirow{2}{*}{ All Patients } & \multicolumn{2}{|c|}{$\begin{array}{c}\text { Non-severe Category } \\
(n=60)\end{array}$} & \multicolumn{4}{|c|}{$\begin{array}{l}\text { Severe Category } \\
(n=30)\end{array}$} \\
\hline & & $\begin{array}{l}\text { Survivor } \\
(\mathrm{n}=59)\end{array}$ & $\begin{array}{c}\text { Non-survivor } \\
(n=1)\end{array}$ & $p$ valuet & $\begin{array}{l}\text { Survivor } \\
(n=12)\end{array}$ & $\begin{array}{c}\text { Non-survivor } \\
(n=18)\end{array}$ & $p$ valuet \\
\hline Creatine kinase & & & & 0.039 & & & 0.016 \\
\hline Median (IQR) - U/liter & $\begin{array}{r}173.6 \\
(83.4-287.3)\end{array}$ & $\begin{array}{r}123.98 \\
(76.0-213.1)\end{array}$ & $\begin{array}{r}463.59 \\
(-)\end{array}$ & & $\begin{array}{r}158.7 \\
(115.0-193.0)\end{array}$ & $\begin{array}{r}178.7 \\
(149.4-276.6)\end{array}$ & \\
\hline $\begin{array}{l}\text { Distribution - no./total no. (\%) } \\
>168 \text { U/liter } \\
\text { Median (IQR) - U/liter }\end{array}$ & $\begin{array}{r}31 / 60(51.7) \\
170.9 \\
(195.2-419.3)\end{array}$ & $\begin{array}{r}15 / 36(41.7) \\
365.68 \\
(210.71754 .2)\end{array}$ & $\begin{array}{r}1 / 1(100) \\
463.59 \\
(-)\end{array}$ & & $\begin{array}{r}4 / 9(44.44) \\
195.20 \\
(187.5-217.6)\end{array}$ & $\begin{array}{r}11 / 14(78.6) \\
248.38 \\
(178.7-330.5)\end{array}$ & \\
\hline $\begin{array}{l}\text { Creatine kinase-MB isoenzyme } \\
\text { Median (IQR) - U/liter }\end{array}$ & $\begin{array}{r}0.6 \\
(0.4-1.3)\end{array}$ & $\begin{array}{r}0.45 \\
(0.30-0.80)\end{array}$ & $\begin{array}{r}21.34 \\
(-)\end{array}$ & 0.079 & $\begin{array}{r}0.50 \\
(0.35-0.85)\end{array}$ & $\begin{array}{r}1.0 \\
(0.80-1.80)\end{array}$ & 0.053 \\
\hline $\begin{array}{l}\text { Distribution - no./total no. (\%) } \\
>25 \text { U/liter } \\
\text { Median (IQR) - U/liter }\end{array}$ & $\begin{array}{r}4 / 63(6.3) \\
274.2 \\
(123.3-431.5)\end{array}$ & $\begin{array}{r}3 / 38(7.9) \\
319.04 \\
(229.2-408.9)\end{array}$ & - & & - & $\begin{array}{r}1 / 13(7.7) \\
74.96 \\
(-)\end{array}$ & \\
\hline $\begin{array}{l}\text { High sensitivity cardiac troponin I } \\
\text { Median (IQR) - ng/ml }\end{array}$ & $\begin{array}{r}0.009 \\
(0.004-0.026)\end{array}$ & $\begin{array}{r}0.006 \\
(0.003-0.01)\end{array}$ & $\begin{array}{r}0.046 \\
(-)\end{array}$ & $<0.001$ & $\begin{array}{r}0.012 \\
(0.005-0.025)\end{array}$ & $\begin{array}{r}0.028 \\
(0.013-0.127)\end{array}$ & $<0.001$ \\
\hline $\begin{array}{l}\text { Distribution - no./total no. (\%) } \\
>0.015 \mathrm{ng} / \mathrm{ml} \\
\text { Median (IQR) - U/liter }\end{array}$ & $\begin{array}{r}27 / 80(33.8) \\
0.043 \\
(0.027-0.185)\end{array}$ & $\begin{array}{r}9 / 49(18.4) \\
0.043 \\
(0.024-0.105)\end{array}$ & $\begin{array}{r}1 / 1(100) \\
0.046 \\
(-)\end{array}$ & & $\begin{array}{r}5 / 12(41.7) \\
0.035 \\
(0.033-0.037)\end{array}$ & $\begin{array}{r}12 / 18(66.7) \\
0.066 \\
(0.029-0.393)\end{array}$ & \\
\hline $\begin{array}{l}{ }^{*} \text { The denominators of patients who } \\
\text { rounding. IQR denotes interquartile ra } \\
\dagger P \text { value less than } 0.05 \text { is considered } \\
\ddagger \text { Data for disposition were missing in }\end{array}$ & $\begin{array}{l}d \text { in the analysis } \\
\text { ses. }\end{array}$ & rovided If the & & & group. Perc & s may not total & ecause 0 \\
\hline
\end{tabular}

\begin{tabular}{|c|c|c|c|c|c|c|c|}
\hline \multirow{2}{*}{ Parameter } & \multirow{2}{*}{ All Patients } & \multicolumn{2}{|c|}{$\begin{array}{c}\text { Non-severe Category } \\
(\mathrm{n}=60)\end{array}$} & \multicolumn{4}{|c|}{$\begin{array}{c}\text { Severe Category } \\
(n=30)\end{array}$} \\
\hline & & $\begin{array}{l}\text { Survivor } \\
(n=59)\end{array}$ & $\begin{array}{c}\text { Non-survivor } \\
(\mathrm{n}=1)\end{array}$ & p valuet & $\begin{array}{l}\text { Survivor } \\
(n=12)\end{array}$ & $\begin{array}{c}\text { Non-survivor } \\
(n=18)\end{array}$ & $p$ valuet \\
\hline \multicolumn{8}{|c|}{ Cardiovascular complications - no./total no. (\%) } \\
\hline New onset or worsening heart failure & $1 / 90(1.1)$ & $1 / 59(1.7)$ & - & - & - & & \\
\hline Significant arrhythmia & $6 / 90(6.7)$ & & - & - & $3 / 12(25.0)$ & $3 / 18(16.7)$ & 0.293 \\
\hline \multicolumn{8}{|l|}{ Type of arrhythmia } \\
\hline Ventricular tachycardia & $2 / 90(2.2)$ & - & - & - & & $2 / 18(11.1)$ & $<0.001$ \\
\hline Arial fibrillation & $4 / 90(4.4)$ & - & - & - & $3 / 12(25.0)$ & $1 / 18(5.6)$ & 0.125 \\
\hline Ectopic atrial rhythm & $1 / 90(1.1)$ & - & - & - & $1 / 12(8.3)$ & & - \\
\hline Vasopressor requiring/shock & $26 / 90(28.9)$ & $2 / 59(3.3)$ & $1 / 1(100)$ & 0.002 & $6 / 12(50.0)$ & 15/18 (83.3) & 0.011 \\
\hline Venous thromboembolism & $2 / 90(2.2)$ & - & - & - & $1 / 12(8.3)$ & $1 / 18(5.6)$ & 0.605 \\
\hline \multicolumn{8}{|l|}{ Other complications - no./total no. (\%) } \\
\hline Acute respiratory distress syndrome & $36 / 90(46.8)$ & $8 / 59(16.3)$ & $1 / 1(100)$ & $<0.001$ & $11 / 12(91.7)$ & $16 / 18(88.9)$ & $<0.001$ \\
\hline Acute kidney injury & $23 / 90(25.6)$ & $8 / 59(13.6)$ & $1 / 1(100)$ & 0.001 & $4 / 12$ (33.3) & $10 / 18(55.6)$ & $<0.001$ \\
\hline Coagulopathy & $3 / 90(3.3)$ & & & - & & $3 / 18(16.7)$ & 0.001 \\
\hline $\begin{array}{l}\text { Physician-diagnosed secondary } \\
\text { infection }\end{array}$ & $21 / 90(23.3)$ & $4 / 59(6.8)$ & $1 / 1(100)$ & $<0.001$ & $6 / 12(50.0)$ & $10 / 18(55.6)$ & $<0.001$ \\
\hline Intubation & $28 / 85(32.9)$ & $5 / 57(8.8)$ & $1 / 1(100)$ & 0.361 & $7 / 9(77.8)$ & $15 / 17(88.2)$ & 0.001 \\
\hline $\begin{array}{l}\text { Presence of primary composite endpoint } \\
\text { - no./total no. }(\%)^{* *}\end{array}$ & $39 / 90(43.3)$ & $8 / 59(13.6)$ & $1 / 1(100)$ & $<0.001$ & $12 / 12(100)$ & $18 / 18(100)$ & $<0.001$ \\
\hline \multicolumn{8}{|c|}{ Clinical outcomes at data cutoff - no./total no. $(\%)^{\dagger \dagger}$} \\
\hline Discharge from hospital & $66 / 85(77.6)$ & $57 / 57(100)$ & - & $<0.001$ & $7 / 12(58.3)$ & - & $<0.001$ \\
\hline Death & $19 / 85(22.4)$ & - & $1 / 1(100)$ & - & -1 & $18 / 18(100)$ & $<0.001$ \\
\hline \multicolumn{8}{|c|}{$\begin{array}{l}\text { * The denominators of patients who were included in the analysis are provided if they differed from the overall numbers in the group. Percentages may not total } 100 \text { because } 0 \\
\text { rounding. IQR denotes interquartile range. } \\
\dagger P \text { value less than } 0.05 \text { is considered significant. } \\
\ddagger \text { The primary composite end point was admission to an intensive care unit, the use of mechanical ventilation, or death. } \\
\text { § Data for disposition were missing in } 5.5 \% \% \text { of cases. }\end{array}$} \\
\hline
\end{tabular}


$\mathrm{ng} / \mathrm{ml}[\mathrm{IQR}, 0.19-2.01]$ vs. $0.12 \mathrm{ng} / \mathrm{ml}[\mathrm{IQR}, 0.06-0.19], p<0.001)$ and non-severe $(0.25 \mathrm{ng} / \mathrm{ml}$ vs. $0.05 \mathrm{ng} / \mathrm{ml}$ [IQR, 0.02-0.12], $p<$ $0.001)$ categories.

\section{CARDIAC BIOMARKERS}

The median tCK level, 173.6 U/liter (IQR, 83.4-287.3) was significantly higher among non-survivors than those who survived across both categories (463.59 U/liter vs. $123.98 \mathrm{U} /$ liter [IQR, 76.0-213.1], $p=0.039)$ and (178.5 U/liter [IQR, 149.4-276.6] vs 158.7 U/liter [IQR, 115.0-193.0], $p=0.016)$.

The median hsTnl for all patients was $0.009 \mathrm{ng} / \mathrm{ml}$ (IQR, 0.0040.026). It was significantly higher among non-survivors than those who survived for both severe $(0.028 \mathrm{ng} / \mathrm{ml}$ [IQR, 0.013$0.127]$ vs. $0.012 \mathrm{ng} / \mathrm{ml}$ [IQR, 0.005-0.025], $p<0.001$ ) and nonsevere cases $(0.046 \mathrm{ng} / \mathrm{ml}$ vs. $0.006 \mathrm{ng} / \mathrm{ml}$ [IQR, 0.003-0.010], $p<0.001$ ). Among those with elevated hsTnl levels, the median was $0.016 \mathrm{ng} / \mathrm{ml}$ [IQR, 0.027-0.185]

No statistical significance for CK-MB isoenzyme levels across both categories was found.

\section{COMPLICATIONS AND CLINICAL OUTCOMES}

Among the non-survivors in the severe category, two (11.1\%) developed ventricular tachycardia. There were four (4.4\%) patients who developed new onset atrial fibrillation in the severe category and only one (5.6\%) did not survive. Among the 26 patients who were in need of vasopressor support in the severe category, there were significantly more non-survivors who required vasopressors than those who survived $(15,83.3 \%$ vs $6,50 \%, p=0.011)$. Also, of the 28 patients who were intubated in this study, $16(57.1 \%)$ died, whereas $12(42.9 \%)$ survived ( $p=$ 0.001).

Thirty-six (46.8\%) individuals developed acute respiratory distress syndrome, and $16(88.9 \%)$ in the severe category did not survive $(p<0.001)$. Acute kidney injury was noted among those who did not survive relative to those who did in the severe category $(10,55.6 \%$ vs. $6,50 \%, p<0.001)$. Among the twentyone $(23.3 \%)$ patients in the severe category who had secondary infections during the course of admission, ten (55.6\%) did not survive while six $(50 \%)$ did $(p<0.001)$. At study cut off, a total of $66(77.6 \%)$ patients were discharged from the hospital improved, while there were $19(22.4 \%)$ who died, one $(5.3 \%)$ in the nonsevere and 18 (94.7\%) in the severe category. The patient in the non-severe category died from brainstem failure secondary to hemorrhagic stroke.

\section{ASSOCIATION OF CARDIAC BIOMARKERS WITH THE} PRIMARY COMPOSITE ENDPOINTS AND DISEASE SEVERITY Table 6 shows the Spearman rho correlation analysis of cardiac biomarkers with the primary composite endpoints and disease severity. There was significant weak positive association between elevated tCK levels and disease severity $(R, 0.251, p=0.017)$; ICU admission ( $R, 0.357, p=0.001)$; need for intubation $(R$, $0.321, p=0.002)$; and in-hospital mortality $(R, 0.313, p=0.003)$. High sensitivity troponin I had significant moderate positive association with in-hospital mortality $(R, 0.434, p=<0.001)$, while it had significant weak positive association between disease severity $(\mathrm{R}, 0.379, p=<0.001)$; ICU admission $(\mathrm{R}, 0.357, p=$ $0.001)$; and intubation $(\mathrm{R}, 0.346, p=0.001)$.

\section{ASSOCIATION OF ELEVATED CARDIAC BIOMARKERS WITH IN-HOSPITAL MORTALITY \\ The binary logistic regression analysis for elevated cardiac biomarkers with in-hospital mortality is shown in Table 7. Univariate analysis showed that elevated levels of tCK and of hsTnl were associated with increased risk of dying. Results of the multivariate regression analysis, showed that the two cardiac biomarkers were independently associated with in-hospital mortality among COVID patients.}

Figure 2 shows the ROC curve for the cardiac biomarkers with in-hospital mortality. It shows that hsTnl was a better predictor for in-hospital mortality than tCK. The ROC, cutoff and AUC of the ROC curve analysis with in-hospital mortality is shown in Table 8. At $0.015 \mathrm{ng} / \mathrm{ml}$ reference value, hs troponin I was a good significant predictor for in-hospital mortality (AUC, 0.829 [95\% $\mathrm{Cl}, 0.735-0.923]), p<0.001)$, while creatine kinase was a poor significant predictor (AUC, 0.677 [95\% Cl, 0.531-0.823], $p=$ $0.018)$ at $168 \mathrm{U} /$ liter.

The optimal cutoff value for hsTnl of $0.010 \mathrm{ng} / \mathrm{ml}$ (J, 0.574; $\mathrm{d}$,

\begin{tabular}{|c|c|c|c|c|c|c|c|c|}
\hline \multirow[t]{2}{*}{ Cardiac Biomarkers } & \multicolumn{2}{|c|}{$\begin{array}{c}\text { Disease Severity } \\
(n=90)\end{array}$} & \multicolumn{2}{|c|}{$\begin{array}{l}\text { ICU Admission } \\
(\mathrm{n}=90)\end{array}$} & \multicolumn{2}{|c|}{$\begin{array}{c}\text { Intubation } \\
(\mathrm{n}=90)\end{array}$} & \multicolumn{2}{|c|}{$\begin{array}{c}\text { Mortality } \\
(\mathrm{n}=90)\end{array}$} \\
\hline & $\mathrm{R}^{*}$ & Sig. ${ }^{\dagger}$ & $\mathbf{R}^{*}$ & Sig. $\dagger^{\dagger}$ & $\mathrm{R}^{*}$ & Sig..$^{\dagger}$ & $\mathbf{R}^{*}$ & Sig. ${ }^{\dagger}$ \\
\hline Creatine kinase & 0.251 & 0.017 & 0.357 & 0.001 & 0.321 & 0.002 & 0.313 & 0.003 \\
\hline $\begin{array}{l}\text { CK-MB isoenzyme } \\
\text { hs Troponin I }\end{array}$ & $\begin{array}{r}-0.033 \\
0.379\end{array}$ & $\begin{array}{r}0.755 \\
<0.001\end{array}$ & $\begin{array}{l}0.029 \\
0.357\end{array}$ & $\begin{array}{l}0.786 \\
0.001\end{array}$ & $\begin{array}{r}-0.028 \\
0.346\end{array}$ & $\begin{array}{l}0.790 \\
0.001\end{array}$ & $\begin{array}{l}0.021 \\
0.434\end{array}$ & $\begin{array}{r}0.848 \\
<0.001\end{array}$ \\
\hline
\end{tabular}

* Correlation coefficient. Interpretation, 0-0.2, very weak; 0.2-0.4, weak; $0.4-0.6$, moderate; $0.6-0.8$, strong; 0.8-1.0, very strong. $\dagger P$ value less than 0.05 is considered significant.

Table 7. Binary Logistic Regression Analysis of the Association of Elevated Cardiac Biomarkers with Mortality Among COVID-19 Patients.

\begin{tabular}{|c|c|c|c|c|c|c|c|c|}
\hline \multirow{2}{*}{ Cardiac Biomarkers } & \multicolumn{4}{|c|}{ Univariate Analysis } & \multicolumn{4}{|c|}{ Multivariate Analysis } \\
\hline & $\operatorname{Exp}(B)$ & & & Sig. ${ }^{*}$ & $\operatorname{Exp}(B)$ & & & Sig. ${ }^{*}$ \\
\hline Creatine kinase & 4.692 & 1.609 & 13.679 & 0.005 & 4.103 & 1.241 & 13.563 & 0.021 \\
\hline CK-MB isoenzyme & 1.259 & 0.124 & 12.840 & 0.846 & 0.830 & 0.054 & 12.776 & 0.894 \\
\hline hs Troponin I & 8.821 & 2.849 & 27.316 & $<0.001$ & 7.899 & 2.430 & 25.675 & 0.001 \\
\hline
\end{tabular}


Figure 2: Receiver Operator Characteristics (ROC) Curve for Cardiac Biomarkers with In-hospital Mortality Among COVID-19 Patients.

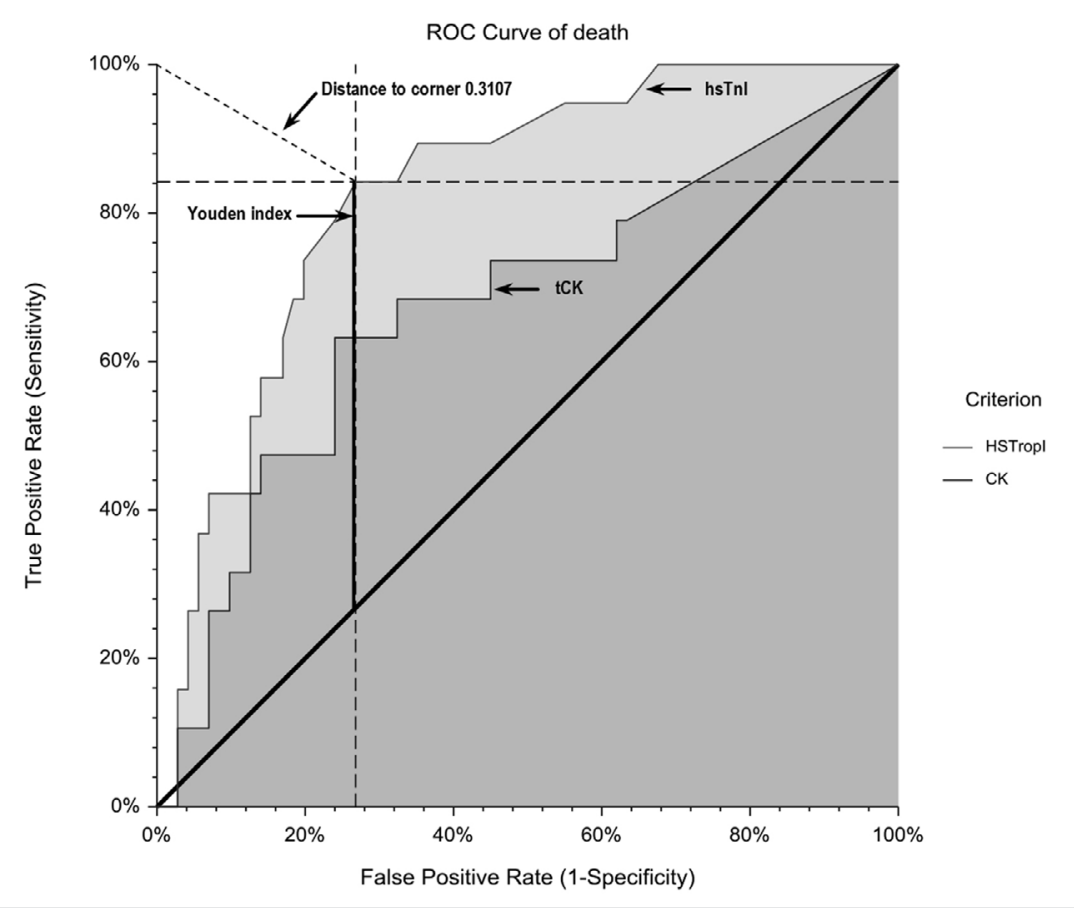

Table 8. ROC Curve, Cutoff and Area Under the Curve Analysis for Elevated Cardiac Biomarkers with In-hospital Mortality Among COVID-19 Patients.

\begin{tabular}{lrr}
\hline & \multicolumn{1}{c}{ hs Troponin I } & \multicolumn{1}{c}{ Creatine kinase } \\
\hline Reference value used & $0.015 \mathrm{ng} / \mathrm{ml}$ & $168 \mathrm{U} /$ liter \\
Area under the Curve (AUC) & $0.829(0.735-0.923)$ & $0.677(0.531-0.823)$ \\
Sig. $^{*}$ & $<0.001$ & $\mathbf{0 . 0 1 8}$ \\
Distance to corner (d) & 0.311 & 0.439 \\
Youden index (J) & 0.574 & 0.392 \\
Derived optimal cutoff value & $0.010 \mathrm{ng} / \mathrm{ml}$ & $173 \mathrm{U} /$ liter \\
Sensitivity (TPR) & $0.842(0.604-0.966)$ & $0.632(0.384-0.837)$ \\
Specificity (TNR) & $0.732(0.614-0.831)$ & $0.761(0.645-0.854)$ \\
False positive rate & $0.268(0.169-0.386)$ & $0.239(0.146-0.355)$ \\
False negative rate & $0.158(0.034-0.396)$ & $0.368(0.163-0.616)$ \\
Positive predictive value (PPV) & $0.457(0.288-0.633)$ & $0.414(0.235-0.611)$ \\
Known prevalence*Adjusted PPV ${ }^{\ddagger}$ & 0.142 & 0.122 \\
Negative predictive value (NPV) & $0.945(0.849-0.989)$ & $0.885(0.778-0.953)$ \\
Known prevalence*Adjusted NPV ${ }^{\ddagger}$ & 0.989 & 0.975 \\
Prevalence & $0.211(0.132-0.310)$ & $0.211(0.132-0.310)$ \\
Accuracy & $0.756(0.654-0.840)$ & $0.733(0.630-0.821)$ \\
Positive likelihood ratio (LR+) & $3.147(2.044-4.844)$ & $2.638(1.540-4.519)$ \\
Negative likelihood ratio (LR-) & $0.216(0.076-0.615)$ & $0.484(0.265-0.885)$ \\
Diagnostics odds ratio & $14.596(3.821-55.766)$ & $5.445(1.850-16.032)$ \\
\hline
\end{tabular}

${ }^{*} P$ value less than 0.05 is considered significant.

$\dagger$ ROC curve analysis was not done for CK-MB isoenzyme since it was not associated with in-hospital mortality

¥Adjusted predictive values based on known prevalence of the disease $(5 \%)$.

\&Definitions adopted from NCSS 2020 Statistical Software (2020). NCSS, LLC. Kaysville, Utah, USA, ncss.com/software/ncss.: ${ }^{37 C u t o f f}$ Value indicates the criterion value range that predicts a positive condition. TPR is the true positive rate or sensitivity is the proportion of the units with a known positive condition for which the predicted condition is positive. TNR is the true negative rate or specificity is the proporion of the units with a known negative condition for which the predicted condition is negative. PPV is the positive predictive value or precison is the proportion of the units with a predicted positive condition for which the true condition is positive. NPV is the negative predictive value is the propolion of the units with a predicted negative condition for which the true condition is notive. Youden Index is the Sensitivity + Specifcity - 1 PPV is the units w a pristis the ratio of the true positive rate (sensitivity) to the false positive rate $(1$ - specificity). LR- is the negative likelihood ratio is he ratio of he false negative rate to the true negative rate (specificity). The diagnostic odds ratio is the ratio of the positive likelihood ratio to the negative likelihood ratio. The distance to the top-left corner of the ROC curve for each cutoff value is given by $d$ $\sqrt{(1-\text { sensitivity })^{2}+(1-\text { specificity })^{2}}$. The accuracy reflects the total proportion of units that are correctly predicted or classified.
0.311) was lower than the reference limit of $0.015 \mathrm{ng} / \mathrm{ml}$ used in this study. It had a corresponding sensitivity of $84 \%$ (TPR, $0.842[95 \% \mathrm{Cl}, 0.604-0.966])$, a specificity of $73 \%$ (TNR, 0.732 [95\% Cl, 0.614-0.831]), accuracy of $76 \%(0.756$ [95\% Cl, $0.654-$ $0.840]$ ), a positive likelihood ratio of 3.147 (95\% Cl, 2.044-4.844) and a negative likelihood ratio of $0.216(95 \% \mathrm{Cl}, 0.076-$ $0.615)$. It had a negative predictive value of hsTnl of 95\% (NPV, 0.945 [95\% Cl, 0.849-0.989]) which increased to $99 \%$ after adjustment to the known prevalence was done (Known prevalence*Adjusted NPV, 0.989).

In contrast, creatine kinase had a larger optimal threshold of $173 \mathrm{U} /$ liter (J, 0.392; $d, 0.439$ ) than the reference limit used in this study of $168 \mathrm{U} /$ liter. It had a sensitivity of $63 \%$ (TPR, 0.632 [95\% Cl,0.384-0.837]) and specificity of $76 \%$ (TNR, $0.761[95 \%$ $\mathrm{Cl}, 0.645-0.854]$ ). After adjustment to the known prevalence of COVID-19, the negative predictive value $89 \%$ (NPV, 0.885 [95\% Cl, 0.778-0.953]) increased to $98 \%$ (Known prevalence*adjusted NPV, 0.975).

\section{DISCUSSION}

Recent epidemiologic and clinical studies of influenza and other acute inflammatory conditions have demonstrated an association of an event leading towards acute coronary syndrome in patients with long-standing coronary artery disease and those with risk factors for atherosclerotic cardiovascular disease, i.e., diabetes, hypertension, dyslipidemia, smoking obesity, inactive lifestyle and significant family history of heart disease. ${ }^{7-12}$ Both severe acute respiratory syndrome coronavirus 2 (SARS-CoV-2), formerly referred to as the 2019 novel coronavirus (2019-nCoV), and the Middle East Respiratory Syndrome coronavirus (MERSCoV) share the same pathobiochemical and biomechanical mechanisms of myocardial damage which increase the difficulty and complexity of patient treatment. ${ }^{13}$

It was also shown that during acute infections, the following risk factors for atherosclerotic cardiovascular disease, like hypertension, cigarette smoking, diabetes mellitus, hyperlipidemia, and obesity, further add to the already heightened risk for acute coronary events among those with long term coronary artery disease. ${ }^{14-16}$

Collated data from China, Italy, and the US 
showed that older individuals with COVID-19 with underlying cardiovascular and other comorbidities, have a higher risk to develop adverse clinical outcomes and to mortality, as compared to those younger and healthier adults. ${ }^{14-17}$

Bonow et al recognized that patients with underlying cardiovascular disease which can be mostly seen in older people had more unfavorable outcomes and death during serious and aggressive inflammatory reactions to COVID-19 compared to younger and healthier individuals. ${ }^{18}$ Likewise, two cohorts demonstrated the association of myocardial injury with increased fatality rate among older patients with preexisting cardiovascular comorbidities and diabetes. ${ }^{19-20}$

Myocardial injury is one of the more clinically significant manifestations of COVID-19. It is not yet well known how this is brought about by SARS-CoV-2 infection due to a lack of randomized controlled trials and the acuteness of this global pandemic. Current data suggest that the interaction between SARS-CoV-2 and cardiac angiotensin-converting enzyme 2 (ACE-2) receptors may precipitate myocardial damage. One of the proposed mechanisms is that cardioprotection provided by angiotensin 1-7 is hindered by the downregulation of ACE-2 receptors thereby resulting in increased inflammatory cytokines such as tumor necrosis factor-alpha (TNFa). ${ }^{21}$ Other hypothesized mechanisms of myocardial injury include induction of interstitial fibrosis in the myocardium by activation of TGF $\beta$ signaling by SARS-CoV-2, exaggerated cytokine response by T-Helper cells (both type 1 and type 2), as well as strong interferon-mediated immune reactions. ${ }^{21}$

These acute coronary events result from the severe increase in myocardial demand triggered by infections that precipitate myocardial injury or type 2 myocardial infarction. ${ }^{14-17}$ Additionally, during severe systemic inflammatory stress, the circulating cytokines released could also lead to atherosclerotic plaque instability and rupture. ${ }^{14-17}$ Wang et al postulated that the increase in metabolic demand brought about by the viral infection potentiates the imbalance and reduce the cardiac reserve thereby making chronic cardiovascular diseases unstable. ${ }^{13}$

Most epidemiologic studies enrolled more severe cases than non-severe ones, ${ }^{8-10}$ but in this study, there were more cases in the non-severe category than in the severe category. The findings in this study of marked elevation of acute inflammatory markers, i.e., neutrophil count, AST, serum ferritin, LDH, creatine kinase, D-dimer, and hs-CRP among subjects with COVID-19 who developed complications were all in congruence with other international and local studies. ${ }^{22-26}$

This study showed similar findings that older patients with co-morbid conditions were predisposed to increase adverse events. ${ }^{12,}$ 26, 28-33 Additionally, it was shown that creatine kinase and hsTnl had a direct association with disease severity and the primary composite endpoints (ICU admission, intubation, and mortality). The strength of correlation was generally weak, except for that of hsTnl with in-hospital mortality where it was moderate. Direct correlations of both tCK and hsTnl with disease severity and the primary composite endpoints were shown in this study. These could be ascribed to the actual myocardial injury caused by the SARS-CoV-19 similar to what was observed in other previous studies. ${ }^{13,15,19,20}$ also demonstrated that the majority of the mortalities have acute myocardial injury as evidenced by increased levels of hsTnl. ${ }^{12,13,15,20,26,34}$ The demonstration of the value of cardiac troponin to in-hospital mortality among COVID-19 patients resembles other previous investigations. 12,13,15,19, 20 Those laboratory-confirmed patients with elevated hsTnl levels were eight times more likely to succumb to the disease relative to those who did not. Also, the odds that COVID patients were more likely to die when they have elevated levels of tCK was increased four times as opposed to those who have normal levels.

The AUC analysis of the ROC curve further showed that hsTnl was a good predictor for in-hospital mortality, while creatine kinase was a poor significant predictor of in-hospital mortality. Evaluating the $95 \% \mathrm{Cl}$ showed that the lower limit of tCK was 0.531 . Anything below the threshold limit of 0.6 would have no value as an instrument, hence nullifying its significance as a tool for predicting in-hospital mortality.

As with other previous studies, the severity of damage to the myocardium was reflected by the increase in hsTnl levels, where the more severe the damage, the more the patient was predisposed to adverse clinical outcome and mortality. ${ }^{12,13,15,19}$, ${ }^{20}$ The extent of insult might affect myocardial function which could draw hemodynamic instability anytime. Hence, the positive association of the biomarker with in-hospital mortality among patients with COVID infection existed.

Studies have shown that high specificity is one characteristic of an extremely good screening test to avoid large numbers of false positives. ${ }^{36-37}$ The cutoff analysis revealed an optimal cutoff value for hsTnl of $0.010 \mathrm{ng} / \mathrm{ml}$ in predicting myocardial injury which was directly associated with in-hospital mortality among COVID-19 patients, and it was specific to as high as $83 \%$, and was sensitive to as high as $97 \%$ at the most. If the test was negative, one can be $99 \%$ confident that there was no myocardial injury resulting from COVID-19 infection. Seventy-six percent of those who will exceed the threshold will be correctly classified as having myocardial damage. The probability of having myocardial injury is increased by $20 \%$ when hsTnl levels exceed $0.010 \mathrm{ng} / \mathrm{ml}$ among COVID-19 patients, whereas it is decreased by $30 \%$ when hsTnl levels are below $0.010 \mathrm{ng} / \mathrm{ml}$.

\section{CONCLUSION}

In this small study of 90 rRT-PCR positive COVID-19 patients with cardiac biomarker determinations, high sensitivity troponin I level is a good tool in significantly predicting in-hospital mortality.

\section{LIMITATIONS}

A much bigger sample size will ensure more robust conclusions. There were missing data on some of the variables, such as symptoms pre-admission (4/90, [4.4\%]) and eventual disposition (5/90, [5.5\%]).

\section{KEYWORDS}

Coronavirus disease-2019. COVID-19. Predictors of In-hospital mortality. Cardiac biomarkers. HS troponin I. ROC analysis. AUC analysis. Cutoff analysis. 


\section{REFERENCES}

1. https://www.worldometers.info/coronavirus/

2. https://Www.who.int/publications/i/item/clinical-management-of-covid-19

3. https://www.idsociety.org/public-health/COVID-19-Resource-Center/ covid19-expanded-resource-center/\#Clinical

4. Wu Z, McGoogan JM. Characteristics of and important lessons from the corona virus disease 2019(COVID-19) outbreak in China: summary of a report of 72314 cases from the Chinese Center for Disease Control and Prevention. JAMA. Published online February 24, 2020. doi: 10.1001/ jama.2020. 26485.

5. ARDS Definition Task Force, Ranieri VM, Rubenfeld GD, Thompson BT, Ferguson ND, Caldwell E, Fan E, Camporota L, Slutsky AS. Acute respiratory distress syndrome: The Berlin Definition. JAMA. 2012 Jun 20;307(23):2526-33. PMID: 22797452.

6. Acute Kidney Injury Work Group. Kidney Disease: Improving Global Outcomes (KDIGO) - Clinical Practice Guideline for Acute Kidney Injury. Kidney Inter. 2012. 2:1-138.

7. https://www.who.int/csr/resources/publications/drugresist/WHO_CDS_ CSR EPH 2002 12/en/

8. Madjid M, Miller CC, Zarubaev VV, et al. Influenza epidemics and acute respiratory disease activity are associated with a surge in autopsyconfirmed coronary heart disease death: results from 8 years of autopsies in 34,892 subjects. EurHeartJ. 2007; 28(10): 1205-1210. doi: 10.1093/ eurheartj/ehm035.

9. Nguyen JL, Yang W, Ito K, Matte TD, Shaman J, Kinney PL. Seasonal influenza infections and cardiovascular disease mortality. JAMA Cardiol. 2016:1(3): 274-281. doi: 10.1001/jamacardio. 2016.04338

10. Kwong JC, Schwartz KL, Campitelli MA, et al. Acute myocardial infarction after laboratory-confirmed influenza infection. N Engl J Med. 2018; 378(4): 345-353. doi:10.1056/ NEJMoa1702090.

11. Huang H, Cai s, Li Y, et al. Prognostic factors for COVID-19 pneumonia progression to severe symptom based on the earlier clinical features: a retrospective analysis. medRxiv Prepr. 2020; 1-3.

12. Hu L, Chen S, Fu Y, et al. Rsisk factors associated with clinical outcomes in 323 COVID-19 in Wuhan, China. medRxiv [Internet]. 2020; 2020.03.25.20037721. Available from: http://medrxiv.org.content/ early/2020/03/26/2020.03.25.20037721. abstract.

13. Wang D, Hu B, Hu C, et al. Clinical characteristics of 138 hospitalized patients with 2019 novel corona virus-infected pneumonia in Wuhan, China. JAMA. 2020;323(11): 1061-1069. doi: 10.1001/jama. 2020.1585.

14. Smeeth $L$, Thomas SL, Hall AJ, Hubbard R, Farrington P, Vallance P. Risk of myocardial infarction and stroke after acute infection or vaccination. N Engl J Med. 2004; 351(25): 2611-2618. doi:10.1056/NEJMoa041747.

15. Huang $C$, Wang $Y$, Li X, et al. Clinical features of patients infected with 2019 novel coronavirus in Wuhan, China. Lancet. 2020;395(10223):497506. doi:10.1016/S0140-6736(20)30183-5.

16. Chen N, Zhou M, Dong X, et al. Epidemiological and clinical characteristics of 99 cases of 2019 novel corona virus pneumonia in Wuhan, China: a descriptive study. Lancet. 2020;395(10223):507513.doi:10.1016/S01406736(20)30211-7.

17. Zhou, P. et al. A pneumonia outbreak associated with a new coronavirus of probable bat origin. Nature https://doi.org/10.1038/ s41586-020-20a12-7 (2020).

18. Bonow R, Fonarow G, O'Gara P, et al. Association of Coronavirus disease 2019 (COVID-19) with myocardial injury and mortality. JAMA Cardiol. Published online March 27, 2020. doi:10.1001/jamacardio.2020.1105
19. Guo T Fan Y Chen M et al. Cardiovascular implications of fatal outcomes of patients with coronavirus disease 2019 (COVID-19). JAMA Cardiol. Published online March 27, 2020. doi:10.1001/ jamacardio.2020.1017.

20. Shi S, Qin M, Shen B, et al. Association of cardiac injury with mortality in hospitalized patients with COVID-19 in Wuhan, China. JAMA Cardiol. Published online March 25, 2020. doi:10.1001/ jamacardio.2020.0950.

21. Shi S, Qin M, Cai Y, Liu T, Shen B, Yang F, Cao S, Liu X, Xiang Y, Zhao $Q$, Huang $H$, Yang B, Huang C. Characteristics and clinical significance of myocardial injury in patients with severe coronavirus disease 2019. European Heart Journal (2020) 0, 1-10. doi:10.1093/eurheartj/ehaa408.

22. Xie J, Hungerford D, Chen $\mathrm{H}$, et al. Development and external validation of a prognostic multivariable model on admission for hospitalized patients with COID-19. medRxiv Prepr. 2020; 1-22.

23. Wu C, Chen X, Cai Y, et al. Risk factors associated with acute respiratory distress syndrome and death in patients with coronavirus disease 2019 pneumonia in Wuhan, China, JAMA Intern Med. 2020; 1-10.

24. Gong J, Ou J, Qiu X, et al. A tool to early predict severe 2019-nove coronavirus pneumonia (COVID-19): a multicenter study using the risk nomogram in Wuhan. medRxiv Prepr. 2020.

25. Huang H, Cai s, Li Y, et al. Prognostic factors for COVID-19 pneumonia progression to severe symptom based on the earlier clinical features: a retrospective analysis. medRxiv Prepr. 2020; 1-3.

26. Liu W, Tao Z, Lei W, et al. Analysis of factors associated with disease outcomes in hospitalized patients with 2019 novel coronavirus disease. China Med J (engl). 2020.

27. Benjamin, CL. Buton-Nabong K, Javier SP. "Biochemical Changes in Coronavirus Disease 2019 (COVID-19) Admitted at a tertiary hospital in Metro Manila: A Retrospective Study." Unpublished manuscript, June 5 2020, typescript.

28. Li K, Chen D, Chen S, Feng Y, Chang C, Wang Z, et al. Radiographic findings and other predictors in adults with COVID-19. medRxiv Prepr. 2020; 2.

29. Zhou F, YuT, du R, et al. Clinical course and risk factors for mortality of adult inpatients with COVID-19 in Wuhan, china: a retrospective study. Lancet [Internet]. 2020;395:1054-62. Available from https://dx.doi. org/10.1016/S0140-6736(20)30566-3.

30. Wu C, Chen X, Cai Y, et al. Risk factors associated with acute respiratory distress syndrome and death in patients with coronavirus disease 2019 pneumonia in Wuhan, China, JAMA Intern Med. 2020; 1-10.

31. Feng Z, Yu Q, Yao S, et al. Early prediction of disease progression in 2019 novel coronavirus pneumonia patients outside Wuhan with CT and clinical characteristics. medRxiv Prepr. 2020; (138)

32. Zeng L, Li J, Liao M, et al. Risk assessment of progression to severe conditions for patients with COVID-19 pneumonia: a single-center retrospective study. medRxiv Prepr. 2020.

33. Chen J, Qi T, Liu L, et al. Clinical progression of patients with COVID-19 in Shanghai, China. J Infect. 2020;80(5):e1-e6.

34. Li Q, Guan X Wu P, et al. Early transmission dynamics in Wuhan, China, of Novel Coronavirus-Infected Pneumonia. N engl J Med 2020; 382:1 199207. DOI: 10.1056/nejmmOA2001316.

35. NCSS 2020 Statistical Software (2020). NCSS, LLC. Kaysville, Utah, USA ncss.com/software/ncss.

Glas, A, Lijmer J, Prins M, Bonsel G, Bossuyt P. The diagnostics odds

36. ratio: a single indicator of test performance. J Clin Epidemiol. 2003 Nov:56(11):1129-35. doi: 10.1016/s0895-4356(03)00177-x.

37. Fischer J, Bachmann, L, Jaeschke R. A readers' guide to the interpretation of diagnostic test properties: clinical example of sepsis. Intensive Care Med (2003) 29:1043-1051 doi: 10.1007/s00134-003-1761-8. 